\section{Lupus erythematosus and lichen planus overlap syndrome:a case report with a rapid response to topical corticosteroid therapy}

\author{
Gulsen Tukenmez Demirci, ${ }^{1}$ \\ Ilknur Kıvanç Altunay, ${ }^{1}$ Sezgi Sarıkaya, ${ }^{1}$ \\ Damlanur Sakiz ${ }^{2}$ \\ 'Dermatology Department and \\ 2Pathology Department, Sisli Etfal \\ Training and Research Hospital, Istanbul, \\ Turkey
}

\section{Abstract}

Lupus erythematosus (LE) and lichen planus (LP) may occur as an overlap syndrome. We report the clinical characteristics of a young man with lesions diagnosed as LE and LP by histopathological and direct immunoflurosence examinations. We achieved remarkable clinical response from the treatment with topical corticosteroids and no recurrence was seen in a 6 months of follow up time. We found this case interesting because of the rapid improvement with corticosteroid and discussed if there is a real overlap or a coexistence according to the literature.

\section{Introduction}

Lupus erythematosus (LE) and lichen planus (LP) are two distinct and well established dermatoses which occasionally can occur as an overlap syndrome. Overlap syndrome is characterized by mixed clinical and histopathological features of both LE and LP. ${ }^{1}$ Although LE and LP are relatively common diseases, an overlap is considered as an uncommon entity. Approximately 50 cases of LE/LP overlap syndrome have been reported in the literature. ${ }^{2}$ However, some authors suggest that most of the cases could be missed as a consequence of its variable clinical and histopathological appearances. ${ }^{2,3}$ We report a case diagnosed as LE/LP overlap syndrome with a rapid improvement to topical corticosteroid treatment. There was no recurrence at 6 months.

\section{Case Report}

A 26-year-old male presented to our outpatient clinic with a 8-months history of persist- ent, erythematous lesions on his back and widely scattered mildly itching papules on upper and lower extremities.

Dermatological examination revealed erythematous, slightly scaly, irregularly bordered, infiltrated large plaques with central atrophy on his back, a butterfly type rash involving nose and malar region and erythema on his ears and neck. (Figure 1A and 1B), The skin of his retroauricular regions were intact. He also had widespread violaceous lichenoid papules on his upper and lower extremites (Figure 1C), but no mucosal or nail involvement was noted.

Laboratory examinations, including complete blood counts, erythrocyte sedimantation rate, routine urine tests were within normal limits except for mild elevations of alanin transaminase and aspartate aminotransferase (44 U/L, $46 \mathrm{U} / \mathrm{L}$; normal limits 0-35 U/L, 045U/L) Antinuclear antibodies, Anti-dsDNA, Anti-ssA, Anti-ssB, Anti-Sm were all negative. Complement C3 and C4 levels were all normal.

Two cutaneous biopsies were taken from a plaque on the back and from the dorsum part of one hand. Histopathology of the biopsy specimen from his back which showed thinning of the epidermis, basal layer vacuolar degeneration, perifollicular chronic inflammatory infiltrates and deposition of mucin in the dermis was consistent with subacute cutaneous LE. Mucin deposition was also shown with alcian blue staining. Direct immunofluorescence (DIF) examination of the plaque lesion revealed deposits of immunoglobulin (IgM> IgG, IgA) and C3 forming a granuler pattern (feature of LE) and linear fibrinogen deposition at the basal membrane zone (BMZ) (feature of LP). (Figure 2) A biopsy specimen from the dorsum of the hand was consistent with the diagnosis of LP with hypergranulosis, with a band-like mononuclear infiltrate at the dermo-epidermal junction (Figure 3 ).

The patient was diagnosed as having LE/LP overlap syndrome after clinical, histopathological and immunohistological examinations. He was treated topically with mometasone furoat $0.1 \%$ cream applied twice daily for two weeks. A rapid improvement of the lesions were seen at the end of the second week.

\section{Discussion}

LE/LP overlap syndrome can be diagnosed with the combination of clinical, histopathological and/or immunopathological features of both diseases in the same patient and/or at the same lesion of one patient. ${ }^{2}$ Histopathological features can be consistent with either LP or LE or both while DIF usually suggests former. ${ }^{4}$ There is still some controversy regarding the definion of this syndrome. It is suggested that true LELP overlap is defined as the presence
Correspondence: Gulsen Tukenmez Demirci, Sisli Etfal Eğitim ve Araştırma Hastanesi Dermatoloji Kliniği, 19 Mayı Cad. Etfal sok. Sisli, 34377 Istanbul, Turkey

Tel. +90.212 .373 .5000 - Fax. +90.212 .353 .5976 .

E-mail: gulsentukenmez@yahoo.com

Key words: cutaneous lupus erythematosus, discoid lupus erythematosus, lichenoid eruption.

Received for publication: 16 June 2011.

Revision received: 4 July 2011.

Accepted for publication: 6 October 2011.

This work is licensed under a Creative Commons Attribution NonCommercial 3.0 License (CC BYNC 3.0).

(C) Copyright G.T. Demirci et al., 2011

Licensee PAGEPress, Italy

Dermatology Reports 2011; 3:e48

doi:10.4081/dr.2011.e48

of LE and LP in the same lesion, whereas the presence of LE features in one lesion and LP features in other one should be considered as a coexistence of LE and LP rather than overlap. ${ }^{5,6}$ Nagao et al. reported a true overlapped LELP patient presenting with single lesion showing combined features of LE and LP which was confirmed by both DIF and histopathological studies. Furthermore, in a study conducted by De Jong et al., with the immunohistochemical examination of the markers for extracellular matrix proteins, it was asserted that LE/LP overlap syndrome can be considered as LP-like LE rather than as a distinct disease. ${ }^{7}$ The lesions on the back in our patient were more consistent with LE clinically and histopathologically,while those on his hands were consistent with LP clinically and histopathologically. DIF taken from his back showed combined immunfluorescence features of LP and LE. In most cases clinical and histopathological features enable us to differantiate between LE and LP, but it may be difficult where as lesions are less typical or an overlapping occurs. Both diseases may show similar histopathological and immunopathological findings of basement membrane changes and colloid bodies. ${ }^{6,8,9}$

As for therapy, topical tacrolimus $0.1 \%$, systemic retinoids and cyclosporine have been reported to be effective in the treatment of this condition. $1,10,11$ We only tried topical corticosteroids. A dramatic improvement occurred in only two weeks. In fact, topical steroids are considered to be inadequate in each disease and systemic therapy is needed. It is interesting to obtain a good response with only topical therapy and also in a relatively short period of 2 weeks in our patient. Follow up seems to be mandatory because the chance of conversion of the syndrome into systemic lupus erythe- 


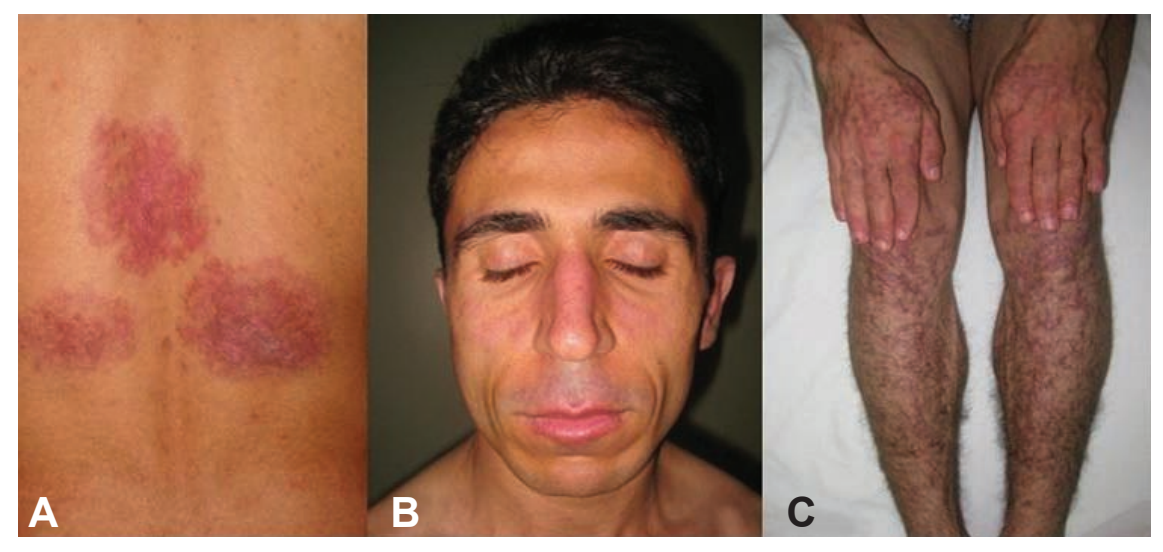

Figure 1. A) Erythematous, slightly, scaly, irregularly bordered, infiltrated three large plaques with central atrophy on the back. B) Butterfly type rash involving the nose and malar region. C) Violaceous lichenoid papules on the upper and lower extremities.

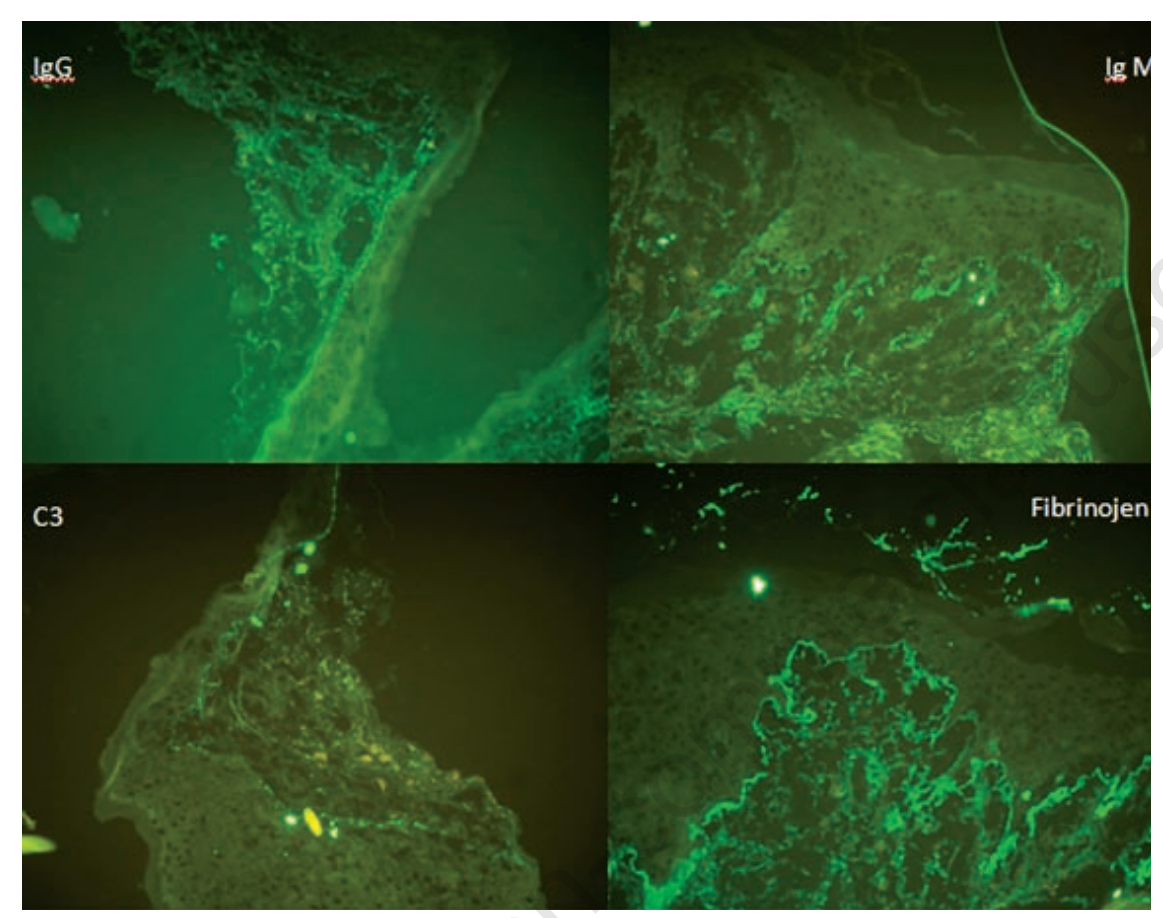

Figure 2. Deposition of Immunoglobulin G, Immunoglobulin M, C3 and fibrinogen in Lupus erythematosus (LE) and lichen planus (LP) lesions on the back has been shown by direct immunofluorescent (Direct immunofluorescence $\times 200$ ).

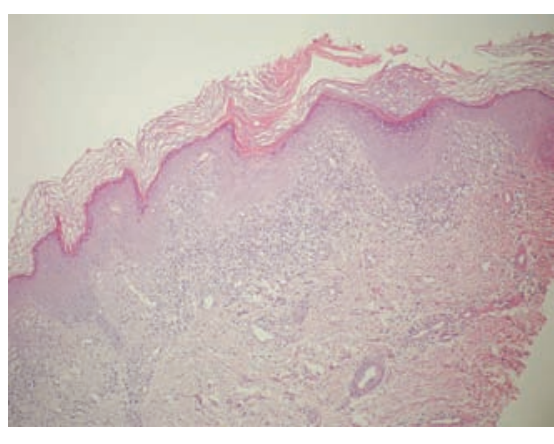

Figure 3. Histopathologic features of the specimen from the dorsum of the hand. The presence band-like mononuclear cell infiltration and hypergranulosis (Hematoxylin and eosin stain $\times 100)$. matosus is reported to be about $5-10 \% .^{12} \mathrm{We}$ did not see any recurrence during 6 months follow-up.

\section{Conclusions}

We report a case which can be identified as LE/LP coexistence depending on the clinical picture. Although deposition of immunoglobulines in a granuler pattern and fibrinogen in a linear pattern at BMZ supports a true overlapping, the lack of cytoid bodies staining with IgM prevents us from suggesting true overlap of LE/LP. Additionally, success of local corticos- teroid therapy alone in this combination of two difficult diseases is another debateble issue. We think it is necessary with more data from more patients to delineate LE/LP overlap and coexistence. It seems that the mistery of the disease continues to remain.

\section{References}

1. Kim H, Pomeranz MK. Lupus erythematosus/lichen planus overlap syndrome. J Drugs Dersmatol;3:311-2.

2. Inaloz HS, Chowdhury MMU, Motley RJ. Lupus erythematosus/lichen planus overlap syndrome with scarring alopecia. J Eur Acad Dermatol Venereol 2001;15:171-4.

3. Mahler V, Hornstein OP, Meyer S, et al. Lupus erythematosus/lichen ruber planus overlap syndrome. 5 cases in a patient sample of the Erlangen University Dermatology Clinic (1894-1895). Hautarzt 1998;49:295-302.

4. James WD, Berger TG, Elston DM. Andrews' Diseases of the Skin: Clinical Dermatology. 10th ed. New York: Elsevier; 2006.

5. Nagao K, Chen KR. A case of lupus erythematosus/lichen planus overlap syndrome. J Dermatol 2006;33:187-90.

6. Lever WH, Schaumburg-Lever G. Histopathology of the Skin, 7th ed. JB Lippincott Company: Philadelphia; 1990. pp 494-505.

7. De Jong EM, Van der Vleuten CJ, Van Vlijmen-Willems IM. Differences in extracellüler matrix proteins, epidermal growth and differentiation in discoid lupus erythematosus, lichen planus and the overlap syndrome. Acta Derm Venereol 1997;77: 356-60.

8. Romero RW, Nesbitt LT Jr, Reed RJ. Unusual variant of lupus erythematosus or lichen planus. Clinical, histopathologic and immunofluorescent studies. Arch Dermatol 1977;113:741-8.

9. Camisa C, Neff JC, Olsen RG. Use of indirect immunofluorescence in the lupus erythematosus/lichen planus overlap syndrome: an additional diagnostic clue. J Am Acad Dermatol 1984;11:1050-9.

10. Grabbe S, Kolde G. Coexisting lichen planus and subacute cutaneous lupus erythematosus. Clin Exp Dermatol 1995;20: 249-54.

11. Tursen $\mathrm{U}, \mathrm{Oz} \mathrm{O}$, Ikizoglu G, et al. A case of lichen planus lupus erythematosus overlap syndrome with eyelid involvement. Eur J Ophthalmol 2002;12:244-6.

12. Jablonska S, Blaszozyk M. Lupus erythematosus. What's new? J Eur Acad Dermatol Venereol 2000;15:103-5. 